\title{
PITÁGORAS E VAN HIELE: UMA POSSIBILIDADE DE CONEXÃO
}

\section{Pythagoras and van Hiele: a possible connection}

José Carlos Pinto Leivas ${ }^{1}$

Resumo: Este trabalho analisa como um grupo de alunos da licenciatura em Matemática e de professores em ação continuada enuncia e representa o Teorema de Pitágoras. A pesquisa tem conotação qualitativa e foi realizada num evento de Educação Matemática, durante a realização de uma oficina sobre os níveis de van Hiele, no segundo semestre do ano de 2010. Aspectos de visualização e representação foram observados, e constatamos que a maioria absoluta dos sujeitos investigados não expressa corretamente o teorema numa linguagem matemática apropriada, estabelecendo conflitos cognitivos entre aspectos de medida e de formas. Além disso, as representações do teorema, feitas pelos sujeitos, se limitam, quando o fazem, a uma única situação, e não dão sinais de identificarem uma generalização do teorema, além do aspecto geométrico de construir quadrados sobre os lados de um triângulo retângulo.

Palavras-chave: Teorema de Pitágoras. Teoria de van Hiele. Educação geométrica. Educação matemática. Formação de professores. Ensino de Matemática. Ensino Superior.

\begin{abstract}
This work analyses how a group of graduate students of Mathematics and practicing teachers represent Pythagoras's Theorem. The research has a qualitative approach and was held at an event of Mathematics Education, during a workshop on the van Hiele levels in the second half of 2010. Aspects of visualization and representation were observed and we found that the majority of investigated subjects don't use an appropriate language for the mathematical theorem, establishing conflict between cognitive aspects and forms of measurement. Furthermore, representations of the theorem, given by the subjects are limited, when they give them, to a single location and they don't give signs to identify a generalization of Theorem beyond the geometric aspect of constructing squares on the sides of a triangle.
\end{abstract}

Keywords: Pythagoras theorem. Theory of van Hiele. Geometric education. Teacher training. Mathematics education. Higher education.

\footnotetext{
${ }^{1}$ Mestrado Profissionalizante em Ensino de Física e de Matemática, Centro Universitário Franciscano de Santa Maria (UNIFRA). Rua Silva Jardim, 1175, Conjunto III, prédio 16, sala 610. Santa Maria, RS, Brasil. 97.010-491. leivasjc@unifra.br
} 


\section{Introdução}

Em nosso entender, o Teorema de Pitágoras é um dos principais assuntos a ser tratado na Escola Básica e, consequentemente, deve ter uma atenção especial nos cursos de formação de professores de Matemática. Segundo nossa vivência como professor de diversos níveis de ensino, não ocorre uma generalização do teorema, o que deixa os estudantes com uma concepção única de como ele se apresenta e se aplica a diversas situações, como é comprovado na pesquisa aqui apresentada.

Com o advento de certos recursos didáticos para o ensino, o estudante da licenciatura em Matemática e, por decorrência, o da Escola Básica, memoriza uma fórmula e uma visualização de quadriculados postados sobre os lados de um triângulo retângulo e cria, na maioria das vezes, obstáculos epistemológicos por envolver e não distinguir aspectos geométricos de aspectos numéricos.

Dizendo de outra forma, o enunciado "A soma dos quadrados das medidas dos catetos é igual ao quadrado da medida da hipotenusa" deixa os estudantes, e professores com a falsa ideia de que "quadrado da medida de um cateto", que é uma questão de medida, é a mesma coisa que "um quadrado", que é geométrica. Por não serem desenvolvidas outras possibilidades de representações geométricas com a mesma conotação numérica anterior, é que se configura o obstáculo anunciado.

Segundo os Parâmetros Curriculares Nacionais (PCN) (BRASIL, 1988), os conteúdos de Matemática destinados ao Ensino Fundamental se apresentam organizados em quatro blocos, sendo dois deles "Espaço e Forma" e "Grandezas e Medidas". No que diz respeito ao primeiro, encontramos: "O trabalho com espaço e forma pressupõe que o professor de Matemática explore situações em que sejam necessárias algumas construções geométricas com régua e compasso, como visualização e aplicação de propriedades de figuras, além da construção de outras relações" (BRASIL, 1998, p. 51).

No que diz respeito ao segundo bloco, o referido documento indica que

Outro conteúdo destacado neste bloco é a obtenção de algumas medidas não diretamente acessíveis, que envolvem, por exemplo, conceitos e procedimentos da Geometria e da Física. Além disso, os conteúdos referentes a grandezas e medidas proporcionarão contextos para analisar a interdependência entre grandezas e expressá-las algebricamente. (BRASIL, 1998, p. 52)

A Geometria, ao longo dos séculos, tem passado por várias fases, sendo uma delas aquela proposta por René Descartes ao reunir geometria e números, de modo que, ao utilizar coordenadas, forma e lugar puderam ser estudados, como não acontecia com a geometria grega de Euclides, e isso proporcionou que o número pudesse ser visualizado geometricamente, de acordo com Mlodinov (2010). Para o autor, "Graças a Descartes, os conceitos geométricos tais como coordenadas e gráficos, senos e cossenos, vetores e tensores, ângulos e curvaturas, aparecem em todos os contextos da física - da eletrônica do estado sólido à estrutura em grande escala [...]" (MLODINOV, 2010, p. 10). 
Pitágoras e Van Hiele: uma possibilidade ...

Dessa forma, acreditamos que promover visualização, na tentativa de eliminar certo obstáculo envolvendo o Teorema de Pitágoras, poderá ser um objeto de pesquisa envolvendo a teoria de van Hiele para o desenvolvimento do raciocínio em Geometria.

\section{Visualização-representação-van Hiele: o que diz a literatura}

A Geometria tem sofrido transformações em seu aspecto estrutural, no seu ensino e, até mesmo, na criação de diversas geometrias. O próprio Kant, em sua obra a Crítica da Razão Pura, por volta de 1780, segundo Mlodinow (2010, p. 123), assim se expressou:

Percebendo que os geômetras daquele tempo apelavam para o senso comum e para figuras gráficas nas suas "demonstrações", acreditou que a pretensão de rigor deveria ser dispensada, e adotada a intuição. Gauss adotou uma posição oposta - o rigor era necessário, e a maioria dos matemáticos era incompetente.

Aspectos de imaginação, intuição e visualização estão intimamente ligados e constituem, atualmente, elementos que podem contribuir para uma educação geométrica e um melhor desempenho em geometrias, segundo nossa forma de conceber a Geometria atual e futuramente. A esse respeito, Arcavi (1999, p. 62) afirma: "Outro papel de visualização em outro contexto "simbólico" é que a solução visual para um problema pode permitir-nos "ver", que é envolver-se com conceitos e significados os quais podem ser facilmente contornados pela solução simbólica do problema”.

No que diz respeito a esses aspectos visuais, o termo teorema visual pode ser dado por uma pequena ou uma ampla definição, segundo Davis (1993). Para ele, na visão ampla, podem ser incluídos todos os resultados do plano elementar e da geometria sólida, que parecem ser intuitivamente óbvios. Exemplifica:

Uma boa parte do plano elementar e da geometria sólida é visualmente óbvia. É fácil tirar conclusões a partir de figuras que têm muitas simetrias. Numerosos teoremas (Pitágoras, por exemplo) podem ser assim apresentados para que conclusões sejam óbvias. Platão sabia disso. Estes são os aha! é de nosso conhecimento. Alguns filósofos da matemática dizem que o objeto de uma boa parte da pesquisa é encontrar maneiras de apresentar o material que se "vê através" para a conclusão. (DAVIS, 1993, p. 336, grifo nosso)

O processo de visualização em Matemática é discutido por Jones (1998), indicando que o mesmo pode ser mental ou físico, e de imaginação, o qual pode ser pictórico. $\mathrm{O}$ autor relaciona imaginação e percepção, imaginação e memória, a natureza de imagens dinâmicas e a interação entre imaginação e desenvolvimento conceitual. Dessa forma, acreditamos que atividades que desenvolvam formas de representação geométrica do Teorema de Pitágoras possam contribuir para tal processo dedutivo formal que, segundo a teoria de van Hiele, é um dos níveis mais avançados de desenvolvimento mental em Geometria. 
Para Piaget e Inhelder (1993), há uma grande dificuldade na análise da psicogênese do espaço, e está relacionada ao fato de a construção das relações espaciais se encontrar em dois planos distintos: o perceptivo ou sensório-motor e o plano representativo ou intelectual. Segundo os autores, H. Poincaré (apud PIAGET; INHELDER, 1993, p. 17)

liga a formação do espaço a uma intuição sensível e relaciona suas vias profundas sobre a significação do grupo dos deslocamentos ao jogo das sensações propriamente ditas, como se o espaço sensório-motor fornecesse o essencial da representação geométrica e como se o intelecto trabalhasse sobre o sensível já previamente elaborado.

Para eles, desde o nascimento, há construção de um espaço sensório-motor ao mesmo tempo em que se desenvolvem a percepção e a motricidade até a chegada à linguagem e à representação figurada, ou seja, quando se chega ao símbolo correspondente. Com isso, "Após, somente após, vem o espaço representativo, cujos inícios coincidem com o da imagem e do pensamento intuitivo, contemporâneos da aparição da linguagem." (PIAGET; INHELDER, 1993, p. 18)

Para esses autores, é o caráter misto entre certas intuições espaciais figuradas, aquelas que se relacionam às formas dos objetos sólidos (o reconhecimento tátil), que ultrapassa a percepção pura e supõe a tradução das percepções táteis e dos movimentos em imagens visuais. Além disso, para eles, a passagem da percepção das formas à sua representação ocorre em crianças de dois a sete anos.

Em reação a dúvidas decorrentes da teoria cognitiva de Piaget e da experiência em sala de aula, o casal van Hiele elabora uma teoria levando em conta o desenvolvimento mental em Geometria dos alunos, segundo Costa (2000), para quem o modelo centra-se em como se desenvolvem as formas particulares de ensino, e não o crescimento de estruturas mentais, contrariamente ao que foi estudado por Piaget.

O casal van Hiele (1959 apud NASSER, 1992, p. 33) descreveu os níveis correspondentes ao modelo de pensamento em Geometria como sendo:

algumas etapas na aprendizagem e curva de aprendizagem, mas, por outro lado, há muitas etapas que não estão relacionadas com esses níveis de pensamento. Essas etapas são resultado do método de ensino utilizado. Os níveis de pensamento são inerentes à elaboração de um pensamento próprio e independente do método empregado, mesmo que o último deva ser levado em conta.

A literatura tem apresentado várias nomenclaturas sobre os níveis. Explicitamos, neste artigo, aquela constante em Van de Walle (2009), conforme a seguir.

. nível 0: visualização - os objetos de pensamento nesse nível são as formas e "o que elas parecem", enquanto os produtos de pensamento são as classes ou agrupamentos de formas que são "parecidas"; 
Pitágoras e Van Hiele: uma possibilidade ...

- nível 1: análise - os objetos de pensamento são as classes de formas, mais do que as formas individuais, e como produtos de pensamentos nesse nível estão as propriedades das formas;

. nível 2: dedução informal - os objetos de pensamento nesse nível são as propriedades das formas, e os produtos de pensamento são as propriedades dos objetos geométricos;

- nível 3: dedução - os objetos do pensamento são as relações entre as propriedades dos objetos geométricos, e os produtos de pensamento são sistemas axiomáticos dedutivos para Geometria;

- nível 4: rigor - os objetos do pensamento são sistemas dedutivos axiomáticos para a Geometria, e os produtos de pensamento são comparações e confrontos entre os diferentes sistemas axiomáticos da Geometria.

Podemos perceber que esses níveis partem da visualização como base fundamental para o desenvolvimento da teoria em apreço, e chegam ao penúltimo nível com a expectativa de que os estudantes consigam estabelecer relações entre os objetos geométricos, como foi o pretendido com a questão de pesquisa.

Neste trabalho, a partir da literatura envolvendo tanto matemática quanto psicologia da aprendizagem matemática, consideramos o conceito de visualização em Matemática proposto por Leivas $(2009$, p. 22), como sendo "um processo de formar imagens mentais, com a finalidade de construir e comunicar determinado conceito matemático, com vistas a auxiliar na resolução de problemas analíticos ou geométricos."

Assim, se indivíduos conseguem ter uma imagem mental do Teorema de Pitágoras a partir de certa representação geométrica, entendemos que se tornarão mais fáceis a interpretação e a relação entre os aspectos geométricos e os algébricos envolvidos nesse teorema, particularmente em sua forma generalizada, e não apenas naquela de uso comum, ou seja, quadrados construídos sobre os lados do triângulo retângulo.

\section{A questão de pesquisa}

Uma motivação para a presente pesquisa consiste no indicativo fornecido pelos PCN (BRASIL, 1988) no que diz respeito às dificuldades que os alunos encontram quanto a articulações entre as propriedades conhecidas por eles e a forma como organizam o conjunto de sua representação, mesmo aqueles que já se encontram no terceiro ciclo. Tais dificuldades também são enfrentadas pelos ingressantes em cursos de licenciatura em Matemática e, até mesmo, pelos futuros professores, em suas práticas em estágio supervisionado, como tivemos oportunidade de verificar em muitas situações de ensino, ministrando essa disciplina.

Levamos em conta, também, a citação a seguir.

As atividades de Geometria são muito propícias para que o professor construa junto com seus alunos um caminho que a partir de experiências concretas leve-os a compreender a importância e a necessidade da 
Leivas, J. C. P.

prova para legitimar as hipóteses levantadas. Para delinear esse caminho, não se deve esquecer a articulação apropriada entres os três domínios citados anteriormente: o espaço físico, as figuras geométricas e as representações gráficas. (BRASIL, 1998, p. 126)

Além disso, fizemos uso do exemplo citado neste documento a respeito dos desvios obtidos sobre essa articulação quanto ao Teorema de Pitágoras, e o uso de quebra-cabeças para comprovar o resultado e a ausência de demonstração, o que é algo comum ao fazermos uso desse recurso didático. Dessa forma, ao planejar e desenvolver, como convidado, um minicurso sobre a teoria de van Hiele, destinada a professores em ação continuada e a alunos de cursos de licenciatura em Matemática, num evento de Educação Matemática, no segundo semestre do ano de 2010, promovido por uma universidade federal, surgiu a seguinte questão de pesquisa: "como os indivíduos concebem o enunciado do Teorema de Pitágoras e qual a forma geométrica concebida por eles sobre o teorema?"

\section{A pesquisa}

A presente pesquisa segue uma abordagem qualitativa. Segundo a definição de Denzin e Lincoln (1994, p. 1), "pesquisa qualitativa é um campo de inquérito próprio. É uma transversalidade, cruzando disciplinas, campos e matérias." Para os autores, qualquer descrição do que constitui pesquisa qualitativa deve trabalhar num certo campo e momento histórico, podendo significar diferentes coisas em cada um desses.

Pesquisadores qualitativos estudam coisas em seu ambiente natural, para dar significado ou interpretar fenômenos em termos dos significados que as pessoas dão a eles. A pesquisa qualitativa envolve o uso de estudo e coleta de uma variedade de materiais empíricos - estudo de caso, experiência pessoal, introspectiva, história de vida, entrevista, observações, históricos e textos visuais - que descrevem momentos problemáticos, de rotina e significados na vida dos indivíduos. (DENZIN; LINCOLN, 1994, p. 2, grifo nosso)

Realizar a pesquisa num evento de Educação Matemática, durante um minicurso oferecido a professores e estudantes da licenciatura em Matemática, em nosso entender, enquadra-a como qualitativa, delimitando os aspectos sociológicos envolvidos. Analisar os aspectos visuais das representações feitas pelos indivíduos é algo que deve ser levado em consideração em uma pesquisa qualitativa, uma vez que, se os indivíduos formarem uma concepção mental de um conceito, então, o mesmo pode ser traduzido facilmente por um desenho, uma gravura ou um símbolo apropriado.

Para Harper (1994, p. 403), "sociologia visual é primariamente um subcampo da sociologia qualitativa - a gravação, análise e comunicação da vida social por meio de fotografias, filmes e vídeo". O autor questiona sobre o que podemos dizer sobre a pesquisa visual para a sociologia hoje e amanhã, e fornece indicadores sobre o que é relevante para se fazer 
pesquisa e ensino sobre visualização, particularizando para os registros fotográficos com os quais podemos associar as representações em Geometria.

A utilização do registro escrito é uma forma produtiva na pesquisa qualitativa, pela facilidade maior do que a entrevista oral ou gravada, uma vez que o indivíduo tem um tempo para a reflexão e análise do seu pensamento. $O$ pesquisador tem melhores condições de analisar e interpretar o texto escrito pelos indivíduos pesquisados. A esse respeito, Hodder (1994, p. 393) afirma:

Apesar da utilidade da distinção entre documentos e registros, a minha preocupação aqui é mais com os problemas de interpretações de textos escritos de todos os tipos. Tais textos são de grande importância para a pesquisa qualitativa, pois, em termos gerais, o acesso pode ser fácil e de baixo custo, porque as informações prestadas podem diferir e não podem ser disponibilizados sob a forma oral, e porque os textos resistem e, portanto, dão uma visão histórica.

Antes de iniciar as atividades correspondentes ao nível 3 da teoria de van Hiele, o da dedução formal, em que os objetos do pensamento são as relações entre as propriedades desses - e esperando que os indivíduos, nesse nível, sejam capazes de apreciar a necessidade de provar, a partir de uma série de argumentos dedutivos -, o pesquisador distribuiu os participantes em grupos de dois ou três, aleatoriamente escolhidos, de acordo com interesse pessoal ou por disposição na sala. A cada grupo forneceu uma folha em branco.

Tendo perguntado se todos conheciam, pelo menos de nome, o Teorema de Pitágoras, e, recebendo resposta afirmativa de todos os participantes, projetou um slide da seguinte forma:

Teorema de Pitágoras: escreva o enunciado desse Teorema.

Solicitou que cada grupo registrasse em forma de texto, em linguagem coloquial, o seu enunciado na folha distribuída, colocando o nome dos participantes e a escolaridade de cada um.

Depois de certo tempo, projetou o seguinte slide:

Teorema de Pitágoras: faça uma representação geométrica do Teorema.

A seguir, o investigador distribuiu envelopes, contendo quebra-cabeças, a fim de que fossem feitas as montagens respectivas - o que não é objeto desta pesquisa -, por meio das duas seguintes solicitações:

a) montar o TANGRAN, com base no que escreveram sobre o Teorema de Pitágoras;

b) o grupo que primeiro montar deve fazer um registro da montagem em seu caderno e chamar o professor.

O objetivo desses questionamentos foi verificar se os participantes tinham a generalização do teorema, objeto do nível 3 da Teoria de van Hiele - dedução formal; e as atividades que se seguiram visaram à compreensão das diversas formas de representação visual-geométrica do Teorema de Pitágoras de forma generalizada.

A fim de preservar a identidade dos participantes, os mesmos foram designados por letras maiúsculas e serão caracterizados em seu ambiente natural de atuação profissional, o Quadro 1. 
Leivas, J. C. P.

Quadro 1. Componentes dos grupos e formação

\begin{tabular}{|c|c|}
\hline Grupos & Participantes - formação \\
\hline 1 & $\begin{array}{l}\text { A - aluna do } 8^{\circ} \text { semestre de licenciatura em Matemática } \\
\text { B - aluna do } 8^{\circ} \text { semestre de licenciatura em Matemática }\end{array}$ \\
\hline 2 & $\begin{array}{l}\text { C - aluna do } 3^{\circ} \text { semestre de licenciatura em Matemática } \\
\text { D - professora do Ensino Superior } \\
\text { E - professor do Ensino Superior }\end{array}$ \\
\hline 3 & $\begin{array}{l}\mathrm{F} \text { - aluna do } 4^{\circ} \text { semestre de licenciatura e do bacharelado em Matemática } \\
\mathrm{G} \text { - professora do Ensino Médio } \\
\mathrm{H} \text { - aluna do } 5^{\circ} \text { semestre de licenciatura em Matemática }\end{array}$ \\
\hline 4 & $\begin{array}{l}\text { I- aluna do } 8^{\circ} \text { semestre de licenciatura em Matemática } \\
\text { J - professora da Escola Básica }\end{array}$ \\
\hline 5 & $\begin{array}{l}\mathrm{K} \text { - aluno do } 5^{\circ} \text { semestre de licenciatura em Matemática } \\
\mathrm{L} \text { - aluno do } 5^{\circ} \text { semestre de licenciatura em Matemática }\end{array}$ \\
\hline
\end{tabular}

Fonte: Elaborado pelo autor

Os dados do Quadro 1 permitem delinear o ambiente em que a pesquisa foi realizada quanto aos sujeitos investigados. De um total de 12 participantes, oito são alunos da licenciatura em Matemática, variando do $3^{\circ}, 4^{\circ}, 5^{\circ}$ e $8^{\circ}$ período; dois são professores universitários, e dois, professores em exercício na Escola Básica.

O Quadro 2 reúne os textos que expressam o enunciado do Teorema de Pitágoras, feitos pelos cinco grupos.

Quadro 2. Enunciados do Teorema de Pitágoras registrados pelos grupos [mantida a escrita original]

\begin{tabular}{|c|c|}
\hline Grupos & \multicolumn{1}{c|}{ Enunciados do Teorema de Pitágoras } \\
\hline $\mathbf{1}$ & O quadrado do lado maior é a soma dos quadrados dos outros 2 lados. \\
\hline $\mathbf{2}$ & $\begin{array}{l}\text { O quadrado construído sobre a medida da hipotenusa é }=\mathrm{a}+\text { dos } \\
\text { quadrados construídos sobre a medida dos catetos. }\end{array}$ \\
\hline $\mathbf{3}$ & $\begin{array}{l}\text { O quadrado da medida da hipotenusa é igual à soma dos quadrados das } \\
\text { medidas dos catetos. }\end{array}$ \\
\hline $\mathbf{4}$ & $\begin{array}{l}\text { Soma dos quadrados dos catetos é igual ao quadrado da hipotenusa. } \\
\text { Num } \Delta \text { retângulo a medida da hipotenusa ao quadrado é igual somas dos } \\
\text { quadrados dos comprimentos dos catetos. }\end{array}$ \\
\hline
\end{tabular}

Fonte: Elaborado pelo autor

Observando o Quadro 2, anterior, percebemos que o grupo 1, formado por dois estudantes de final da Licenciatura, não distingue, em sua formulação, a questão de medida da geométrica, uma vez que, ao enunciar "quadrado do lado maior", está misturando esses dois 
aspectos e, da mesma forma, com a segunda parte do texto. Além disso, a escrita realizada não expressa o desejado para o nível da maioria dos indivíduos respondentes.

Quanto ao enunciado registrado pelo grupo 2, formado por dois professores do Ensino Superior, constatamos uma falta de cuidado com a linguagem empregada, pois mistura a língua materna com a simbólica matemática. O enunciado nos conduz a interpretar que os participantes associam ao Teorema de Pitágoras apenas o fato geométrico adquirido do senso comum, a saber, uma álgebra geométrica constituída por um quadrado cuja área é equivalente à soma das áreas de outros dois quadrados, não exatamente expressa de forma apropriada. Notamos, entretanto, a exemplo do que ocorre com o grupo 1, falta de diferenciação entre medidas e formas, pois o enunciado caracteriza um quadrado construído sobre a medida da hipotenusa, o que é impossível matematicamente.

O grupo 3 apresenta um enunciado matematicamente correto, ao explicitar a forma numérica "o quadrado da medida da hipotenusa". Até onde podemos perceber, o grupo, que é formado por um professor do Ensino Médio e por estudantes de períodos distintos, tem uma visão do Teorema pelas medidas, e não pelas formas.

Quanto ao grupo 4, formado por um estudante da licenciatura em Matemática e uma professora da Escola Básica, traz o enunciado sem distinguir objeto geométrico de objeto numérico, podendo aí se estabelecer um obstáculo à aprendizagem do Teorema, já que enuncia "quadrados dos catetos" e "quadrado da hipotenusa", sendo que cateto é um objeto geométrico, e, com isso, a visualização obtida pelos alunos sobre o Teorema é única, aquela construindo quadrados sobre os lados de um triângulo retângulo. Usualmente, essa forma leva os estudantes a obterem a comprovação do Teorema, elevando ao quadrado a medida dos catetos, e não elaborando a álgebra geométrica, como já dito antes no artigo.

O grupo 5, formado por estudantes do $5^{\circ}$ período da licenciatura, tem uma boa percepção da diferença entre os aspectos geométricos e numéricos, uma vez que enuncia corretamente "medida da hipotenusa ao quadrado". Explicita, também, no enunciado, a validade de a relação existir no triângulo retângulo. Apenas mistura a simbologia matemática com a linguagem materna no enunciado, o que não é matematicamente conveniente.

Com base na análise feita nos enunciados do Teorema de Pitágoras, pelos participantes da pesquisa, podemos concluir que, para a maioria deles, não há clareza na distinção entre aspectos geométricos e numéricos, ou seja, não há maior preocupação com o que os PCN (BRASIL, 1988) denotam nos dois blocos: Geometria e Formas; Grandezas e Medidas, deixando antever um conflito cognitivo a ser formado nos futuros professores quanto aos dois aspectos envolvidos na pesquisa - até onde vão os aspectos geométricos e os de medida?

Partiremos agora para a segunda parte da pesquisa realizada, ou seja, analisar a questão apresentada: "Faça uma representação geométrica do Teorema de Pitágoras".

O grupo 1 apresentou a representação constante da Figura 1; o grupo 2 apresentou a representação constante da Figura 2; o grupo 3 apresentou a representação constante da Figura 3; o grupo 4 apresentou a representação constante da Figura 4 e o grupo 5 apresentou a representação constante da Figura 5. 
Leivas, J. C. P.

Figura 1. Representação geométrica do grupo 1

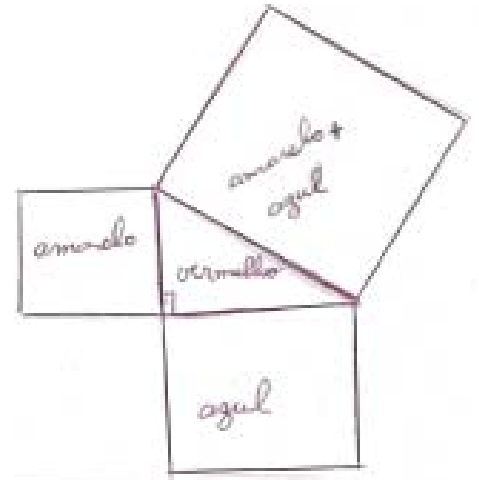

Fonte: Dados da pesquisa

Figura 3. Representação geométrica do grupo 3

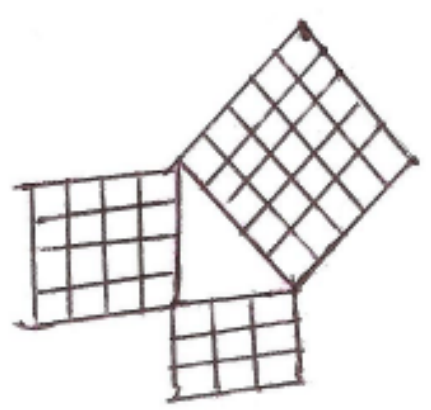

Fonte: Dados da pesquisa
Figura 2. Representação geométrica do grupo 2

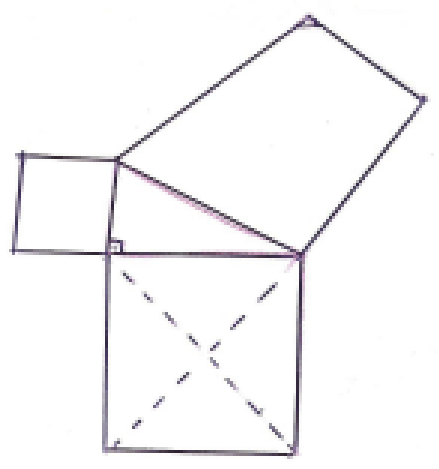

Fonte: Dados da pesquisa

Figura 4. Representação geométrica do grupo
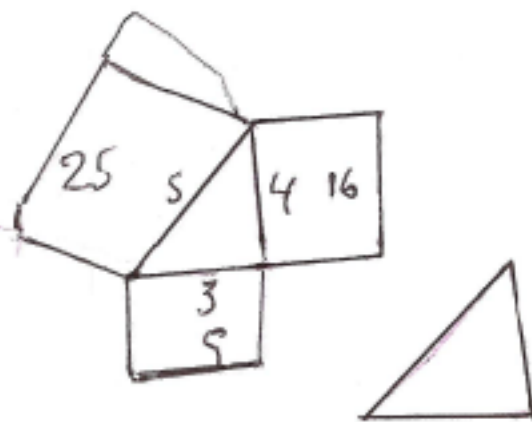

Fonte: Dados da pesquisa

Figura 5. Representação geométrica do grupo 5
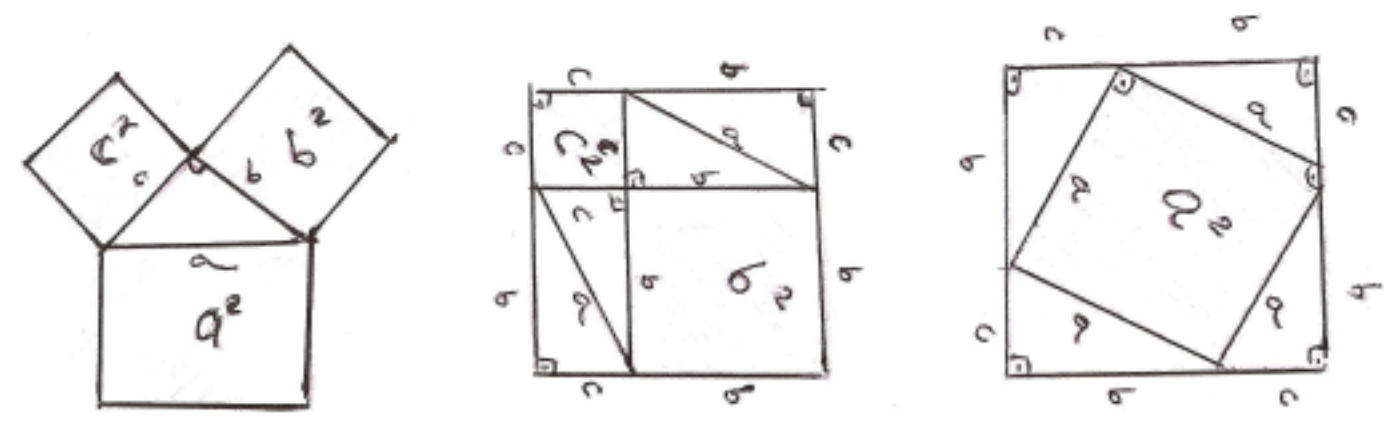

Fonte: Dados da pesquisa 
A partir das representações feitas, podemos deduzir que os quatro primeiros grupos têm como representação geométrica do Teorema de Pitágoras aquela forma comum e única trabalhada na licenciatura em Matemática, e, por consequência, na Escola Básica, ou seja, a formulação algébrica ou numérica e sua simples aplicação memorística, além da representação geométrica, por meio de quadrados construídos sobre os lados do triângulo retângulo. Dessa forma, a falta de desenvolvimento de habilidades mentais visuais na formação inicial dos indivíduos pode ser observada. Tais habilidades, se fossem desenvolvidas na formação inicial, conforme Leivas (2009), auxiliariam na construção e comunicação de conceitos, bem como favoreceriam a resolução de problemas diversos.

A representação feita pelo grupo 3, em um quadriculado, trazendo a ideia de área, talvez seja resultado do que usualmente consta em livros didáticos. Notamos que, nesse grupo, há participação de uma professora do Ensino Médio. Percebemos, assim, que o ensino inicial sobre aspectos visuais está presente nos indivíduos, necessitando, entretanto, se generalizarem tais aspectos de modo que as habilidades mentais visuais não sejam perdidas no decorrer da escolaridade e, sim, desenvolvidas.

De forma similar, o grupo 4, que tem uma professora da Escola Básica como participante, sugere, em sua representação, uma terna pitagórica, que é outra forma usualmente empregada em livros didáticos para tratar do tema, ou seja, a relação algébrica já citada anteriormente, inclusive, utilizando terna de números inteiros. Vemos, com isso, que, ao utilizar tais ternas, não há um crescimento nas estruturas mentais dos indivíduos, que naturalmente deveriam partir para outras medidas e representações geométricas.

Quanto às representações feitas pelo grupo 5, verificamos existir um avanço em relação às anteriores, uma vez que invocou certa demonstração visual do teorema. Tais representações são, em nosso entender, importantes na construção de conceitos, inclusive, os do Teorema de Pitágoras em suas diversas representações geométricas.

Observamos, ainda, a falta de cuidado nas representações apresentadas por todos os grupos. Visualmente, é perceptível que as representações não são de quadrados, e isso pode ser decorrência da falta de hábito de construções e, particularmente, na questão de medidas, um dos destaques apresentado neste trabalho. De certa forma, isso corrobora as dificuldades apontadas por Piaget e Inhelder (1993).

\section{Conclusão}

Considerando que todo teorema em Matemática tem uma demonstração, que toda demonstração tem uma ponto de partida, ou seja, as condições iniciais ou hipóteses, e que toda demonstração tem por objetivo comprovar ou validar certa propriedade para todos os casos, a partir dessas condições iniciais, a formulação de um enunciado correto na mente do professor e dos estudantes da licenciatura em Matemática é fundamental tanto ao ensino quanto à aprendizagem. Assim, demonstrar que o Teorema também vale para outras figuras construídas sobre os lados de um triângulo retângulo, em nosso entender, deveria constar da formação inicial do futuro professor de Matemática.

A utilização de aspectos visuais, seja qual for o recurso usado pelo professor em sala de aula, nos parece um excelente indicativo de como iniciar o processo dedutivo em Matemá- 
tica, e isso é corroborado pela teoria de van Hiele, a qual inicia, em seu primeiro nível, o da visualização, e constrói essa teoria de desenvolvimento de raciocínio em Geometria. Ao chegar ao quarto nível, o da dedução formal, é esperado que os indivíduos já tenham atingido um grau de desenvolvimento que lhes permita admirar e sentir a necessidade de uma demonstração.

Do que foi possível observar na tentativa de criar um enunciado do Teorema de Pitágoras, a pesquisa comprovou que os indivíduos envolvidos, embora quatro deles já sejam formados, não atingiram tal nível de maturidade matemática no conteúdo focado, pois sequer esboçaram algum tipo de ensaio que nos levasse a concluir que tinham algum conhecimento da validade do Teorema em casos mais gerais, o que é esperado, pelo menos, para professores universitários que participaram da pesquisa.

Portanto, ainda há muito a fazer em Educação Geométrica, para que possamos atingir um patamar aceitável no ensino e na aprendizagem de Geometria nos diversos níveis de escolaridade. Acreditamos que desenvolver habilidades de visualização que permitam diversas formas de representação de um conceito matemático é fundamental para se atingir esse objetivo e, especialmente, distinguir Geometria e Formas de Grandezas e Medidas.

\section{Referências}

ARCAVI, A. The role of visual representations in the learning of mathematics. In: ANNUAL MEETING OF THE NORTH AMERICAN CHAPTER OF THE INTERNATIONAL GROUP FOR THE PSYCHOLOGY OF MATHEMATICS EDUCATION, 21., Cuernavaca, Mexico, 1999. Proceedings... Columbus: ERIC Clearinghouse for Science, 1999. p. 55-80.

BRASIL. Ministério da Educação. Parâmetros curriculares nacionais: matemática. Brasília: Secretaria de Educação Fundamental, 1988.

COSTA, C. Visualização, veículo para a educação em geometria. In: ENCONTRO DE INVESTIGAÇÃO EM EDUCAÇÃO MATEMÁTICA, 9., Fundão, Portugal, 2000.

Actas... Lisboa: Sociedade Portuguesa de Ciências da Educação, 2000. p. 157-184.

DAVIS, P. J. Visual theorems. Educational Studies in Mathematics, Dordrecht, v. 24, n. 4, p. 333-344, 1993.

DENZIN, N. K.; LINCOLN, Y. S. Introduction: entering the field of qualitative research. In: __ (Ed.). Handbook of qualitative research. Thousand Oaks: Sage, 1994. p. $1-17$.

HARPER, D. On the authority of the images: visual method at the crossroads. In: DENZIN, N. K.; LINCOLN, Y. S. (Ed.). Handbook of qualitative research. Thousand Oaks: Sage, 1994. p. 403-412. 
Pitágoras e Van Hiele: uma possibilidade ...

HODDER, T. The interpretation of documents and material culture. In: DENZIN, N. K.; LINCOLN, Y. S. (Ed.). Handbook of qualitative research. Thousand Oaks: Sage, 1994. p. 393-402.

JONES, K. Visualization, imagery and the development of geometrical reasoning. In: MEETING OF GEOMETRY WORKING GROUP OF THE BRITISH SOCIETY FOR RESEARCH INTO LEARNING MATHEMATICS, 1998. Informal Proceedings... 1998. p. 123-128. Disponível em: <www.bsrlm.org.uk/IPs/ip18-12/BSRLM-IP-18-12-21.pdf>. Acesso em: 15 jan. 2008.

LEIVAS, J. C. P. Imaginação, intuição e visualização: a riqueza de possibilidades da abordagem geométrica no currículo de cursos de licenciatura de matemática. 294 f. 2009. Tese (Doutorado em Educação) - Universidade Federal do Paraná, Curitiba, 2009.

MLODINOV, L. A janela de Euclides: a história da geometria, das linhas paralelas ao hiperespaço. São Paulo: Geração, 2010.

NASSER, L. Using the van Hiele theory to improve secondary school geometry in Brazil. 1992. Tese (Doutorado em Educação) - London King's College, University of London, London, 1992.

PIAGET, J.; INHELDER, B. A representação do espaço na criança. Porto Alegre: Artes Médicas, 1993.

VAN DE WALLE, J. A. Matemática no Ensino Fundamental: formação de professores e aplicação em sala de aula. 6. ed. Porto Alegre: Artmed, 2009. 